\title{
sciendo
}

\section{The Effect of Neurofeedback Training on the Visual Processing Efficiency in Judo Athletes}

\author{
by \\ Adam Maszczyk1, Paweł Dobrakowski², Magdalena Nitychoruk ${ }^{1}$, Marcin Żak \\ Magdalena Kowalczyk ${ }^{1}$, Michal Toborek ${ }^{3}$
}

\begin{abstract}
This study aimed to analyse the effect of neurofeedback (NFB) training based on beta-wave amplification and theta-wave inhibition on the visual processing efficiency of judo athletes. The study examined 12 male athletes from the national team of the Polish Judo Association. Participants were divided into the experimental $(E G, n=6)$ and the control group (CG, $n=6)$. The NFB training protocol was performed and recorded using a Deymed Truscan system with 24 active channels. The effect of NFB training was examined by computer-based simple and complex reaction tests and selected tests of the Vienna Test System (VST). One - way ANOVA showed statistically significant differences between the CG and the EG in theta and beta values after the first and the second cycle of training. There were statistically significant differences between the $C G$ and the $E G$ in the results of reaction speed tests after individual cycles of training. The highest reduction in simple reaction time was obtained after the second training cycle, when training was performed every second day and lasted four minutes.
\end{abstract}

Key words: EEG, biofeedback, judo athletes, concentration, reaction time, visual processing.

\section{Introduction}

Neurofeedback training (NFB) is becoming increasingly popular. It is used to train the brain to regulate itself, and to help the person training to understand when their brain is in the desired state. NFB is effective in the treatment of ADHD (Arns et al., 2013; Dobrakowski and Lebecka, 2019; Gevensleben et al., 2010; Lansbergen et al., 2011; Lévesque et al., 2006), ADD (Arns et al., 2009; Thompson and Thompson, 1998), and epilepsy (Lubar and Bahler, 1976; Tan et al., 2009). NFB is a technique also used to achieve success in professional sports. NFB is based on feedback, which means that changes in the brain are monitored in real time and can be controlled and modified through training (Thompson, 2003). NFB training is considered an essential element

supporting the preparation of athletes, especially in sports such as shooting and archery (Landers et al., 1991), speed skating (Harvey et al., 2011), gymnastics (Zaichkowsky, 1983), judo (Blumenstein and Orbach, 2012c; Maszczyk et al., 2018), canoeing (Blumenstein and Bar-Eli, 1998), soccer (Wilson et al., 2006), swimming (Bar-Eli and Blumenstein, 2004), windsurfing (Blumenstein and Orbach, 2012d) and dancing (Raymond et al., 2005).

This study aimed to analyse the effect of NFB training based on beta-wave amplification and theta-wave inhibition on the visual processing efficiency of judo athletes. An attempt was also made to develop optimal duration of NFB training aimed at regulation of the level of concentration

\footnotetext{
1 - Institute of Sport Sciences, The Jerzy Kukuczka Academy of Physical Education in Katowice, Poland.

2 - Psychology Institute, Humanitas University in Sosnowiec, Poland.

3 - Department of Biochemistry and Molecular Biology, Miller School of Medicine, University of Miami, USA.
} 
and improving visual attention in judokas, leading to shortening their reaction times.

\section{Methods}

\section{Participants}

The study examined 12 male athletes from the national team of the Polish Judo Association aged from 22 to 25 years. The study sample was chosen purposefully from the national team of the southern region of Poland. Athletes were randomly divided into two subgroups: experimental $(\mathrm{EG}=6)$ and control $(\mathrm{CG}=6)$. All participants were informed about the objectives and procedures used in the study, as well as about the possibility to withdraw from the experiment at any stage. All participants were healthy and refrained from using drugs, alcohol and stimulants (caffeine, energy drinks) 12 hours before each test and training session. The study protocol was approved by the Bioethics Committee of the Jerzy Kukuczka Academy of Physical Education in Katowice, Poland.

\section{Research procedures}

The research was carried out in two cycles. The first cycle included 15 training sessions held every other day. The duration of a training session was 10 minutes. The second part of research, which took place after a 4-week break, was characterised by the same frequency of training sessions, but reduced duration (4 minutes). The basic training protocol in the experimental group was beta1/theta training, aimed to increase concentration and to achieve the so-called narrow attention by athletes.

The tests in the control group followed the same procedures as the experimental group and were characterized by the same duration and frequency of NFB training sessions. The procedure for the training sessions was the same for both groups, but in the control group, instead of the beta1/theta protocol, EEG simulation was performed, regardless of the patterns of brain waves produced by the participant.

Before the beginning of the first cycle of sessions and after the end of each subsequent cycle, tests of simple speed reaction and complex speed reaction were performed in both groups.

Neurofeedback training protocol

The NFB training protocol was performed and recorded using a Deymed Truscan system with 24 active channels (soft. version 6.34.1761, Czech Republic). The device accuracy was confirmed by ISO and CE medical certificates. Before the EEG signal was recorded, the electrodes impedance level and the inter-electrodes level were checked each time using a built-in impedance sensor. The sampling frequency was $1024 \mathrm{~Hz}$. A 50 $\mathrm{Hz}$ main filter, as well as high and low pass filters (1 and $40 \mathrm{~Hz}$ respectively), were used. The reference electrode was placed on the ear. The precondition for starting the diagnosis and NFB training was to achieve an impedance level below $5 \mathrm{k} \Omega$ and measurement between electrodes differing from each other by no more than $1 \mathrm{k} \Omega$.

Each training session in individual cycles was preceded by a 3-min one-channel EEG diagnosis with three reference leads. During this time, the participant was asked to perform the following tasks: to remain seated with eyes open for one minute, to remain seated with eyes closed for one minute, to remain seated with eyes open with an additional activation task of counting backwards from 100 by sevens. During the diagnosis, the reference electrode was placed on the left earlobe, the ground electrode was placed on the right earlobe, and the active electrode at point $\mathrm{Cz}$, according to the 10-20 international system. Then, we were looking for 1-s epochs free from artefacts. EEG parts containing artefacts were excluded from further analysis. Spectral analyses with a fast Fourier transform (FTT) were conducted for artefact-free 1-second epochs allowing for calculation of the mean and standard deviation for each channel for absolute band power, relative band power, median-power frequency and peak-power frequency. Spectral analyses were conducted for the theta and beta frequency bands, $4-7.5 \mathrm{~Hz}$ and $13-30 \mathrm{~Hz}$, respectively. During NFB training, the active electrode was placed at the $\mathrm{C} 3$ point, which made it possible to achieve the main goal of training, that is, to shape the ability to maintain an optimal balance between the activity of fast waves (beta) and slow waves (theta), associated with athletes' performance and concentration. During each NFB session, the percentage of time above the threshold, which is the primary measure of the participant's progress, was also monitored to optimize the level of training difficulty for each participant.

Each EEG recording was assessed by a neurologist certified by the Polish Society of Clinical Neurophysiology, who was blinded for 
participants. Recordings were first analyzed to exclude signals indicating epilepsy. Afterwards, 1$\mathrm{s}$ artefact-free epochs were searched. The recorded signals were filtered between 2 and $40 \mathrm{~Hz}$. An expert visually checked all signals, and periods during which no artifacts were recorded were selected manually and further analysed.

The feedback signal based on the activity recorded from the $\mathrm{C} 3$ electrode was provided in a visual and auditory form. During training, participants were asked to perform a task that consisted of controlling the images displayed on the computer screen so that the plane shown was flying all the time. The plane was moving when appropriate conditions were met at the same time: theta $(4-7.5 \mathrm{~Hz})$ and beta2 band amplitudes (20-30 $\mathrm{Hz}$ ) below a set threshold and beta1 amplitude (13$20 \mathrm{~Hz}$ ) above a set threshold. The effective movement of the plane was accompanied by an amplifying acoustic signal. The thresholds were adjusted manually. The goal for the settings was as follows: the enhance band was set to reinforce $60 \%$ of the time, theta inhibit was true $20 \%$ of the time and beta2 inhibit was true $10 \%$ of the time.

Visual reaction time tests

The effect of NFB training on the visual reaction time in judo athletes was examined by computer-based simple and complex reaction tests and selected tests of the Vienna Test System (VST). The tests were performed in the morning, under conditions conducive to focusing attention on the tasks performed. All tests were repeated twice at 5min intervals, and the better result of the two measurements was registered for further analysis.

The computer test of the speed of the simplest reaction to visual stimuli depended on the fastest possible pressing of a specific key on the keyboard with the right or left hand at the moment when a bright square appeared on the monitor screen. The task that evaluated the complex reaction speed, however, required the fastest possible pressing of a key on the keyboard corresponding to the position of a square appearing on the monitor, which was different in the case of its location in the right, left or central point of the screen. In both tests, the signal appeared 10 times at intervals of $2-6 \mathrm{~s}$. The period from the moment the stimulus appeared until the moment the key was pressed was measured with the accuracy of $0.01 \mathrm{~s}$.

A simple response to visual stimuli was examined using a device for the measurement of reaction speed (RG) being a component of the Vienna Test System. The task of the examined athlete was to move his hand from the so-called "rest key" as fast as possible and to press the "reaction key" when the yellow diode was on. Based on the data obtained, the program calculated the median of reaction speed, reaction time and simple movement time in ms. Complex reaction speed was measured by means of a decision tester (DG), with the test requiring the fastest possible pressing of the appropriate key, depending on the colour of the diode that was on when the stimulus appeared. The program counted the total number of all correct reactions, incorrect reactions, mean reaction speed and standard deviation of mean reaction speed. The signal appeared 15 times.

\section{Statistical Analysis}

The following descriptive statistics were used to evaluate the level of analysed variables: arithmetic mean, standard deviation and coefficient of variation.

The data were tested for homogeneous and normal distribution by means of the Shapiro-Wilk test. Differences between the experimental and control groups were analysed using one-way ANOVA and post-hock Tukey tests. Intragroup differences were examined using the Student's ttest. The level of significance was set at $p<0.05$. The $F$ statistic and the level of significance were evaluated. We reported partial eta square ( $\eta 2)$ values, enabling comparisons of effect sizes in analyses of variance even when there were different numbers of variables (0.01 - small; 0.06 medium and 0.14 - large). Calculations were performed using the STATISTICA 12.0 software (Statsoft).

\section{Results}

One-way ANOVA showed no statistically significant differences between the CG and the EG in the results of the diagnostic tests with eyes open, eyes closed and concentration, and between the CG and the EG in the results of the computer reaction speed test and the Vienna test prior to each cycle of training sessions.

One-way ANOVA with repeated measures showed statistically significant differences between the CG and the EG in beta values after the first and the second cycle of training $\left(F=5.116, p=0.034, \eta^{2}=0.02 ; F=10.463\right.$, 
$p=0.004, \eta^{2}=0.21$, respectively), as well as in theta values after the second cycle of training $(\mathrm{F}=7.647$, $\left.p=0.011, \eta^{2}=0.09\right)$. The differences were not found in the theta values after the first cycle $(\mathrm{F}=3.188, p$ $\left.=0.088, \eta^{2}=0.008\right)$. Figures 1 and 2 present graphical differences between the CG and the EG in the beta and theta values after the first and second training cycles.

There were statistically significant differences between the $C G$ and the EG in the results of the computer and Vienna reaction speed tests after individual cycles of training. In simple reaction tests, the values were as follows: for computer tests: $\mathrm{F}=6.770, p=0.016$ (after the first cycle, $\eta^{2}=0.05$ ); $F=27.120, p=0.003$ (after the second cycle, $\left.\eta^{2}=0.23\right)$; for the Vienna test: $F=$ 5.878, $p=0.024$ (after the first cycle, $\eta^{2}=0.03$ ); $F=$ 41.317, $p=0.002$ (after the second cycle, $\eta^{2}=0.25$ ).

As presented in Tables 1 and 2, analysis of diffrences in reaction speed before and after biofeedback sessions using the Student's t-test in the EG showed statistically significant changes in the simple speed reaction in the computer and Vienna tests.

Figures 3 and 4 present graphical differences in the test results obtained for simple speed reaction before and after biofeedback training sessions [ms].

\begin{tabular}{|c|c|c|c|c|c|}
\hline \multicolumn{3}{|c|}{$\begin{array}{l}\text { Table } \mathbf{1} \\
\text { Results of the Student's t-test used to determine the significance } \\
\text { of changes in simple speed reaction in the computer test [ms]. }\end{array}$} & \multicolumn{3}{|c|}{$\begin{array}{l}\text { Table } \mathbf{1} \\
\text { ermine the significance } \\
\text { e computer test [ms]. }\end{array}$} \\
\hline Variables & $\bar{x}$ & & SD & $p$ & $\eta^{2}$ \\
\hline Before the first cycle & 0.194 & & 0.012 & \multirow{3}{*}{0.004} & \multirow{3}{*}{0.19} \\
\hline & & & & & \\
\hline After the first cycle & 0.186 & & 0.009 & & \\
\hline Before the second cycle & 0.191 & & 0.012 & \multirow[b]{2}{*}{0.002} & \multirow[b]{2}{*}{0.24} \\
\hline After the second cycle & 0.170 & & 0.008 & & \\
\hline \multicolumn{6}{|c|}{$\begin{array}{l}\text { Table } 2 \\
\text { Results of the Student's t-test used to determine the significance } \\
\text { of changes in simple speed reaction in the Vienna test [ms]. }\end{array}$} \\
\hline Variables & & $\bar{x}$ & SD & $p$ & $\eta^{2}$ \\
\hline Before the first cycle & & 0.224 & 0.005 & \multirow[b]{2}{*}{0.001} & \multirow[b]{2}{*}{0.26} \\
\hline After the first cycle & & 0.218 & 0.005 & & \\
\hline Before the second cycle & & 0.220 & 0.006 & \multirow[b]{2}{*}{0.001} & \multirow[b]{2}{*}{0.28} \\
\hline After the second cycle & & 0.207 & 0.003 & & \\
\hline
\end{tabular}




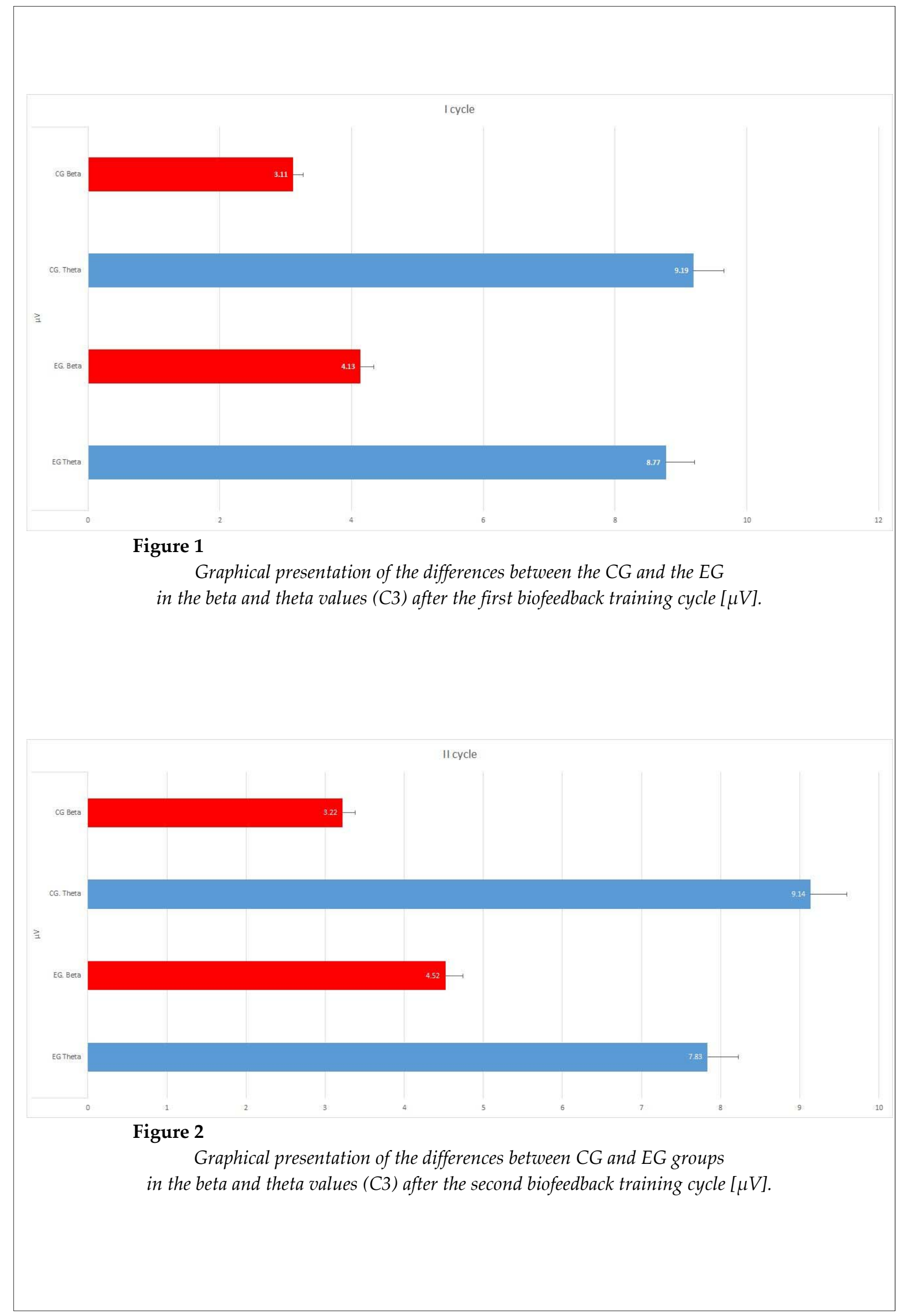




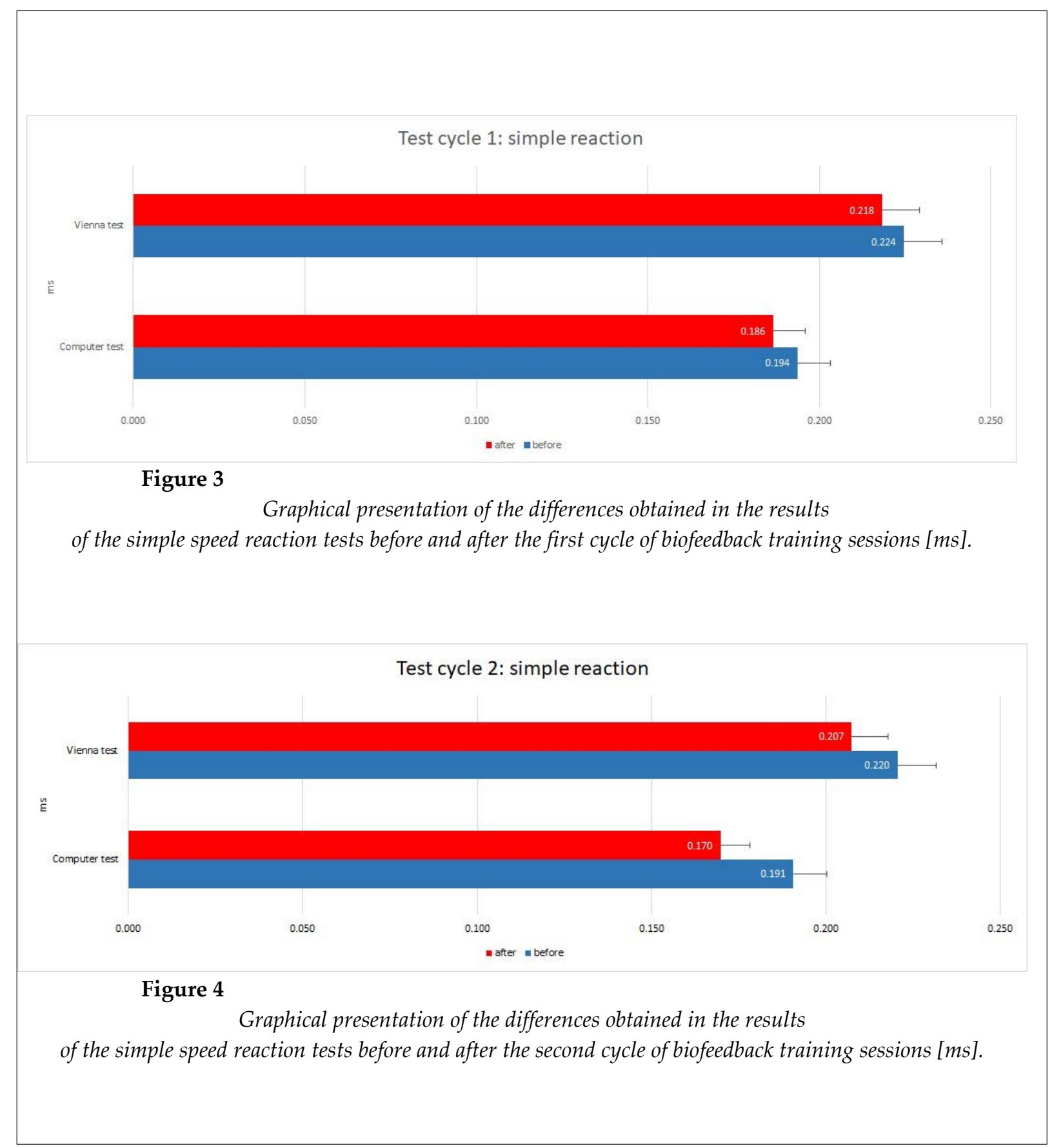

\section{Discussion}

Analysis of numerous scientific reports found that NFB training may have a positive effect on normalization of pathological brain activity accompanying various psychological and neurological disorders. There are significant data on NFB training used in the treatment of ADHD (Arns et al., 2013; Gani, 2009; Gevensleben et al., 2010; Lansbergen et al., 2011), ADD (Arns et al., 2009; Thompson and Thompson, 1998), epilepsy (Lubar and Bahler, 1976; Tan et al., 2009), general anxiety disorders, insomnia, and alcohol abuse (Yucha and Montgomery, 2008). Many publications provide information about factors that determine the effect of NFB training on athletes. However, the literature lacks precisely defined variables for the NFB training methodology.

The main aim of this study was to analyse the effects of the NFB training protocol on the visual reaction speed in judo athletes and to determine the optimal duration of training sessions. The results of the study are consistent 
with some previous findings, which have proved that NFB training reduces the reaction time in athletes. This study indicates that the experimental group showed a statistically significant reduction in reaction time following training compared to the control group. The reduced reaction time may be attributable to the fact that judo athletes learnt to control brain wave activity during NFB training, which positively influenced concentration and speed reaction.

In a study conducted in athletes from the Canadian Speed Skating National Team preparing for the Vancouver Olympic Games, where NFB training was part of their training routine, the most significant improvement in reaction time was observed between the fourth and the fifth (last) training week, with the frequency of two training sessions per week $\mathrm{F}(1,9)=5679.2, p=.001$ (Harvey et al., 2011).

In our study performed in a group of judo athletes, the highest reduction in simple reaction time was obtained after the second training cycle, when training was performed every second day and lasted four minutes. It is interesting why the improvement after the second cycle of training was more pronounced. In our opinion, several factors could have contributed to such results:

- pioneering impact of the first cycle of training (most neurofeedback practitioners emphasize that clinical effects are only visible from the $7^{\text {th }}$ to $8^{\text {th }}$ training session),

- shorter training duration (4 vs. $10 \mathrm{~min}$ ) where it was easier to keep full attention,

- athletes from the national team of the Polish Judo Association, who trained to keep concentration for 4 minutes during each round.

Findings concerning the volume and the number of NFB training sessions needed to produce the desired effects are rather contradictory. Several studies have shown that NFB requires a long-term process (Blumenstein et al., 2012, 2012a; Gani et al., 2008; Logemann et al., 2010), and is not always successful. No statistically significant changes were found following sixteen training sessions (Logemann et al., 2010). In that well-controlled study, however, movie feedback was chosen and we already know that such a stimulus can significantly affect the effectiveness of training (Blumenstein et al., 2012, 2012a). Also, the instructions for participants could have been more precise. A study by Doppelmayr and Weber (2011) demonstrated no changes in reaction time using beta1/theta after 30 training sessions. The response time improved following SMR (sensorimotor rhythm) training at point $\mathrm{C} 4$. Training on the Theta / Beta ratio did not induce significant changes (although the authors did not specify whether on point $\mathrm{C} 3$ or $\mathrm{C} 4$ ).

There are also scientific reports on the use of a relatively small number of NFB sessions which led to statistically significant changes. According to Egner and Gruzelier (2001), ten training sessions were sufficient to learn the beta1/theta protocol. The findings published by Vernon et al. (2003) showed that significant difference using the SMR protocol was achieved following eight sessions only. NFB training was conducted over a period of 4 weeks, with two training sessions per week.

Another study indicated long-term clinical improvement following neurofeedback training regardless of the observed effect on EEG variables (Dobrakowski and Lebecka, 2019).

The discrepancies in the length, frequency and the number of training sessions needed to achieve training objectives depend primarily on individual differences, which in turn point to the need to develop personalised NFB interventions.

An innovative aspect of this study was the participation of selected athletes from the Polish National Judo Team, the use of multidimensional analysis of variance and the placebo group, which helped eliminate the Hawthorne effect and assess in more detail the effectiveness of NFB training in elite athletes.

Introducing a passive control group and combining NFB training with other relaxation techniques would enhance the strength of this study, allowing for comparison of the effect of the NFB training sessions. This issue needs to be considered in future studies using the abovementioned factors.

Results of several studies have confirmed that the NFB method should be successfully used not only as an extension of the standard training process, but in many cases, it can also become its integral part (Blumenstein et al., 2012, 2012a; Lansbergen et al., 2011; Maszczyk et al., 2018; Yucha and Montgomery, 2008). NFB training may significantly improve the reaction time in athletes. The results of the present research may be of great importance for strength and conditioning in sports where quick and precise reactions determine the final success. 


\section{Acknowledgements}

This work was supported by the Ministry of Science and Higher Education of Poland under the Grant NRSA4 04054 and the Institute of Sport Sciences, The Jerzy Kukuczka Academy of Physical Education in Katowice.

\section{References}

Arns M, de Ridder S, Strehl U, Breteler M, Coenen A. Efficacy of neurofeedback treatment in ADHD: The effects on inattention, impulsivity and hyperactivity: A meta-analysis. Clin Eeg Neurosci, 2009; 40: 180189

Arns M, Heinrich H, Strehl U. Evaluation of neurofeedback in ADHD: the long and winding road. Biol psychol, 2013; 95: 108-115

Bar-Eli M, Blumenstein B. Performance enhancement in swimming: The effect of mental training with biofeedback. J Sci Med Sport, 2004; 7: 454-464

Blumenstein B, Orbach I. The road to Olympic medal. In W. A. Edmonds, \& G. Tenenbaum (Eds.). Case studies in applied psychophysiology: Neurofeedback and biofeedback treatments for advances in human performance, 2012; 120-132

Blumenstein B, Bar-Eli M. Self-regulation training with biofeedback training in elite canoers and kayakers' In V. Issurin (Ed.). Science and practice of canoe/kayak high performance training, 1998; 124-132

Blumenstein B, Orbach, I. Biofeedback training at sea' In W. A. Edmonds, \& G. Tenenbaum (Eds.). Case studies in applied psychophysiology: Neurofeedback and biofeedback treatments for advances in human performance, 2012a; 134-143

Dobrakowski P, Lebecka G. Individualized Neurofeedback Training May Help Achieve Long-Term Improvement of Working Memory in Children With ADHD. Clinical EEG and Neuroscience, 2019; Oct 3:1550059419879020. doi: 10.1177/1550059419879020. [Epub ahead of print]

Doppelmayr M, Weber E. Effects of SMR and Theta/Beta Neurofeedback on Reaction Times, Spatial Abilities, and Creativity. Journal of Neurotherapy, 2011; 15(2): 115-129

Egner T, Gruzelier J. Learned self-regulation of EEG frequency components affects attention and event-related brain potentials in humans. Neuroreport, 2001; 12(18): 4155-4159

Gani C, Birbaumer N, Strehl U. Long term effects after feedback of slow cortical potentials and of Theta/Betaamplitudes in children with Attention Deficit Hyperactivity Disorder (ADHD). Int J Bioelectro, 2008; 10(4): 209-232

Gevensleben H, Holl B, Albrecht B, Schlamp D, Kratz O, Studer P, Heinrich H. Neurofeedback training in children with ADHD: 6-month follow-up of a randomised controlled trial. Eur Child Adoles Psy, 2010; 19: 715-724

Harvey R, Beauchamp M, Saab M, Beauchamp P. Biofeedback Reaction-Time Training: Toward Olympic Gold. Biofeedback, 2011;39(1): 7-14

Landers D, Petruzzello S, Salazar W, Crews D, Kubitz K, Gannon T, Han M. The influence of electrocortical biofeedback on performance in pre-elite archers. Med Sci Sport Exer, 1991; 23: 123-129

Lansbergen M, van Dongen-Boomsma M, Buitelaar J, Slaats W. ADHD and EEG-neurofeedback: a doubleblind randomized placebo-controlled feasibility study. J Neural Transm, 2011; 118(2): 275-284

Lévesque J, Beauregard M, Mensour B. Effect of neurofeedback training on the neural substrates of selective attention in children with attention-deficit hyperactivity disorder: A functional magnetic resonance imaging study. Neurosci Lett, 2006; 394: 216-221

Logemann H, Lansbergen M, Van Os T W, Bocker K, Kenemans J. The effectiveness of EEG-feedbackon attention, impulsivity and EEG: A sham feedback controlled study. Neurosci Lett, 2010; 479(1): 49-53

Lubar J, Bahler W. Behavioral management of epileptic seizures following EEG biofeedback training of the sensorimotor rhythm. Biofeedback and Self-reg, 1976; 1(1): 77-104

Maszczyk A, Gołaś A, Pietraszewski P, Kowalczyk M, Cięszczyk P, Kochanowicz A, Smółka W, Zając A. Neurofeedback for the enhancement of dynamic balance of judokas. Biol. Sport, 2018; 35: 99-102 
Raymond J, Sajid I, Parkinson L, Gruzelier J. Biofeedback and dance performance: A preliminary investigation. App Psychoph Biof, 2005; 30(1): 65-73

Tan G, Thornby J, Hammond D, Strehl U, Canady B, Arnemann K, Kaiser D. Meta-analysis of EEG biofeedback in treating epilepsy. Clin EEG Neurosci, 2009; 40: 173-179

Thompson L, Thompson M. Neurofeedback combined with training in metacognitive strategies: Effectiveness in students with ADD. App Psychophys Biof, 1998; 23: 243-263

Vernon D. Can neurofeedback training enhance performance? An evaluation of the evidence with implications for future research. App Psychophys Biof, 2005; 30(4): 347-364

Wilson V, Peper E, Moss D. "The Mind Room" in Italian soccer training: The use of biofeedback and neurofeedback for optimum performance. Biofeedback, 2007; 34(3): 79-81

Yucha C, Montgomery D. Evidence-based practice in biofeedback and neurofeedback, Association for Applied Psychophysiology and Biofeedback, Las Vegas; 2008

Zaichkowsky L. The use of biofeedback for self-regulation of performance states. The mental aspects of gymnastics, 1983; 95-105

\section{Corresponding author:}

\section{Magdalena Nitychoruk}

Institute of Sport Science,

The Jerzy Kukuczka Academy of Physical Education in Katowice, Poland;

40-065 Katowice, Mikolowska 72a str.

E-mail: magda.aneta.krawczyk@gmail.com 